\title{
EVOLUCIÓN DE LAS APORTACIONES HÍDRICAS EN UNA CUENCA DE MONTAÑA DEL SISTEMA CENTRAL: CABECERA FLUVIAL DEL RÍO TORMES (1941-2004)
}

\section{A. CEBALLOS BARBANCHO y E. MORÁN TEJEDA}

Departamento de Geografía, Universidad de Salamanca

C/ Cervantes, s/n. 37002 Salamanca

Correo electrónico de contacto: ceballos@usal.es

RESUMEN. El presente trabajo analiza la tendencia temporal de las aportaciones hídricas en la cabecera fluvial del río Tormes y su relación con la evolución de las principales variables climáticas (temperaturas y precipitaciones) y los cambios ocurridos en la extensión del bosque. Los resultados muestran una disminución de las aportaciones fluviales, con un alto grado de dependencia respecto a las precipitaciones $\left(r^{2}=0,708\right.$; valor-p $\left.<0,001\right)$. A pesar de que el bosque haya aumentado su superficie en un $30 \%$ en lo últimos 45 años, el análisis de las variables hidroclimáticas normalizadas no muestra indicios de que este hecho haya repercutido en una disminución de los aportes hídricos. Un resultado de gran interés ha sido el cambio detectado en la distribución de las aportaciones mensuales debido a la disminución de las precipitaciones en los meses de febrero y marzo, y al notable aumento de las temperaturas en el mes de marzo, que han provocado que el régimen fluvial de la cuenca haya perdido su carácter nival en las últimas décadas.

ABSTRACT. The present work analyses the temporal trend of water discharge in the headwater of the Tormes catchment related with the temporal behaviour of main climatic variables (temperature and precipitation) and the changes that have occurred in the forest cover. The results show how water discharge decreases with a significant correlation with precipitation $\left(r^{2}=0,708\right.$; $p$-value $\left.<0,001\right)$. Although the forest cover has increased about $30 \%$ in the last 45 years, the analysis of hydro-climatic normalized variables does not suggest that the forest expansion has influenced in the discharge decreasing trend. An interesting result has been the monthly variation in discharge due to rainfall decreasing in February and March, and a significant increase in temperature during March. This fact has triggered losses of the snow contribution within the fluvial regime of the catchment in the last decades. 
Palabras clave: aportaciones hídricas, cabecera fluvial, temperaturas, precipitaciones, bosque, río Tormes.

Key words: water discharge, headwaters, temperature, precipitation, forest, Tormes river.

Enviado el 15 de agosto de 2006

Aceptado el 19 de octubre de 2006

\section{Introducción}

Diversos estudios publicados en las últimas décadas manifiestan una creciente preocupación por la disponibilidad presente y futura de los recursos hídricos, ya que el agua es un recurso natural clave para cualquier sociedad con una serie de funciones tanto utilitarias como ambientales (Parry et al., 2000; PNUMA, 2000; IPCC, 2001; Milly et al., 2005). De forma general, los recursos hídricos de un determinado territorio dependen de la interacción de tres tipos de factores: la gestión del agua, el clima, y los usos y/o cobertura del suelo.

En primer lugar, la gestión del agua es clave ya que afecta a la cantidad y calidad de las demandas (Iglesias et al., 2005). Desde un punto de vista técnico debe garantizarse el abastecimiento urbano más el de sectores como el turismo y la agricultura que dependen directamente de los recursos hídricos. Este factor es clave en países con clima mediterráneo y con prácticas agrícolas, en muchos casos insostenibles, que consumen el $80 \%$ del total del agua gastada y con una demanda del recurso concentrada en los meses estivales, cuando la disponibilidad es menor.

En el caso del clima, la evolución que experimenten variables como precipitaciones, temperatura y evapotranspiración es determinante para evaluar la disponibilidad hídrica de un espacio por su papel clave en cualquier balance de agua (Dunne y Leopold, 1978). Hace una década Loaiciga et al. (1996) ya apuntaron un incremento de la variabilidad del ciclo hidrológico a escala global, inducida por el calentamiento del planeta, que debería obligar a muchos países a incrementar sus recursos en políticas de prevención para el control de crecidas, almacenamiento y abastecimiento de agua. Un espacio regional como Europa puede considerarse como representativo de los cambios registrados a escala global en el estudio de la conexión entre procesos climáticos e hidrológicos (Shorthouse y Arnell, 1999), con un marcado contraste entre la Europa septentrional (atlántica) y la Europa meridional (mediterránea). En el caso de Europa septentrional trabajos como los de Arnell (1998) en Gran Bretaña o Kiely (1999) en Irlanda muestran que las precipitaciones están aumentando, así como el caudal de los ríos. En el ámbito geográfico de los países nórdicos, Xu y Halldin (1997) también aprecian un aumento en los caudales, vinculado a mayores precipitaciones, aunque en este caso el comportamiento del sistema sea algo más complejo debido a la importancia de las precipitaciones en forma de nieve. En Europa meridional, destacarían los estudios de Mimikou et al. (2000) en Grecia o el publicado por Ayala-Carcedo y Iglesias (2000) para España, que señalan un descenso tanto en las lluvias como en las aportaciones de ríos y embalses, acompañado siempre de una apreciable irregularidad tanto espacial como temporal en ambas variables. 
Si el clima es determinante para comprender la hidrología de una determinada región, para entender los recursos hídricos relacionados con la producción de escorrentía no puede obviarse el papel que desempeña el área tributaria (Gupta y Waymire, 1990; Loaiciga et al., 1996), especialmente en lo referido a los usos del suelo y cobertura del mismo. Al respecto abundan los trabajos realizados en pequeñas cuencas experimentales que relacionan los valores observados de coeficientes de escorrentía con los usos del suelo (García-Ruiz y Gallart, 1997), que sin embargo en muchos casos carecen de series de datos largas y por tanto insuficientes para detectar tendencias a medio plazo. Uno de los procesos que más interés está despertando en la comunidad científica está siendo la recuperación de las masas forestales en detrimento de las tierras agrícolas y ganaderas como consecuencia del abandono del medio rural y las políticas de la UE, y cómo este hecho está influyendo en la disponibilidad hídrica de los ecosistemas. Una masa forestal densa siempre favorecerá la reducción de la escorrentía que se produce debido a la interceptación por la masa foliar o la propia demanda de agua de los árboles (Rambal, 1987; Joffre y Rambal, 1993; Zhang et al., 2001; Andréassian, 2004; Llorens et al., 2005). Por ejemplo, en el caso de nuestro país, análisis generales como el de Gallart y Llorens (2002) advierten que el decrecimiento observado en las aportaciones de las grandes cuencas ibéricas no se explica únicamente por los cambios ocurridos en las precipitaciones y por el aumento de la demanda de agua para riego, subrayando el papel clave que la recuperación del bosque está desempeñando en las cabeceras fluviales en las últimas décadas. En este sentido, Beguería et al. (2003) estiman una reducción del $30 \%$ en las aportaciones de las cuencas de cabecera pirenaicas entre 1945 y 1995 debido a la recuperación de las masas forestales.

Debido al interés creciente de este tipo de análisis en nuestro país, y a la recomendación planteada por Gallart y Llorens (2002) de analizar la estabilidad temporal de los aportes en régimen natural en donde la serie de datos en las cuencas de cabecera lo permitan, el presente trabajo tiene como objetivos analizar la evolución temporal de la serie histórica de caudales en el curso alto del río Tormes y relacionar la evolución de los mismos con el comportamiento de las principales variables climáticas (temperaturas y precipitaciones) y los cambios experimentados en la cobertura vegetal.

La selección de la cuenca alta del río Tormes se debe a que dispone una serie de datos de caudal suficientemente larga y continua en el tiempo, y al hecho de que el río no haya sido afectado por ninguna obra de regulación y por tanto su comportamiento pueda ser considerado como representativo de un régimen natural.

\section{2. Área de estudio}

El río Tormes es uno de los principales afluentes del Duero por su margen izquierda, con un recorrido de $247 \mathrm{~km}$ y una superficie de drenaje de $7.100 \mathrm{~km}^{2}$ en la última estación de aforos (Embalse de La Almendra) antes de su confluencia. La primera estación de aforos, correspondiente a su cabecera fluvial en la cara norte de la Sierra de Gredos en el Sistema Central, es la de Hoyos del Espino (Ávila), localizada a 40²0'47’ N y $5^{\circ} 11$ ' 47 'O. Este punto nos permite delimitar una cuenca con un área vertiente de $83 \mathrm{~km}^{2}$ (Fig. 1) y una altitud que oscila entre los 2224 y $1250 \mathrm{~m}$. 


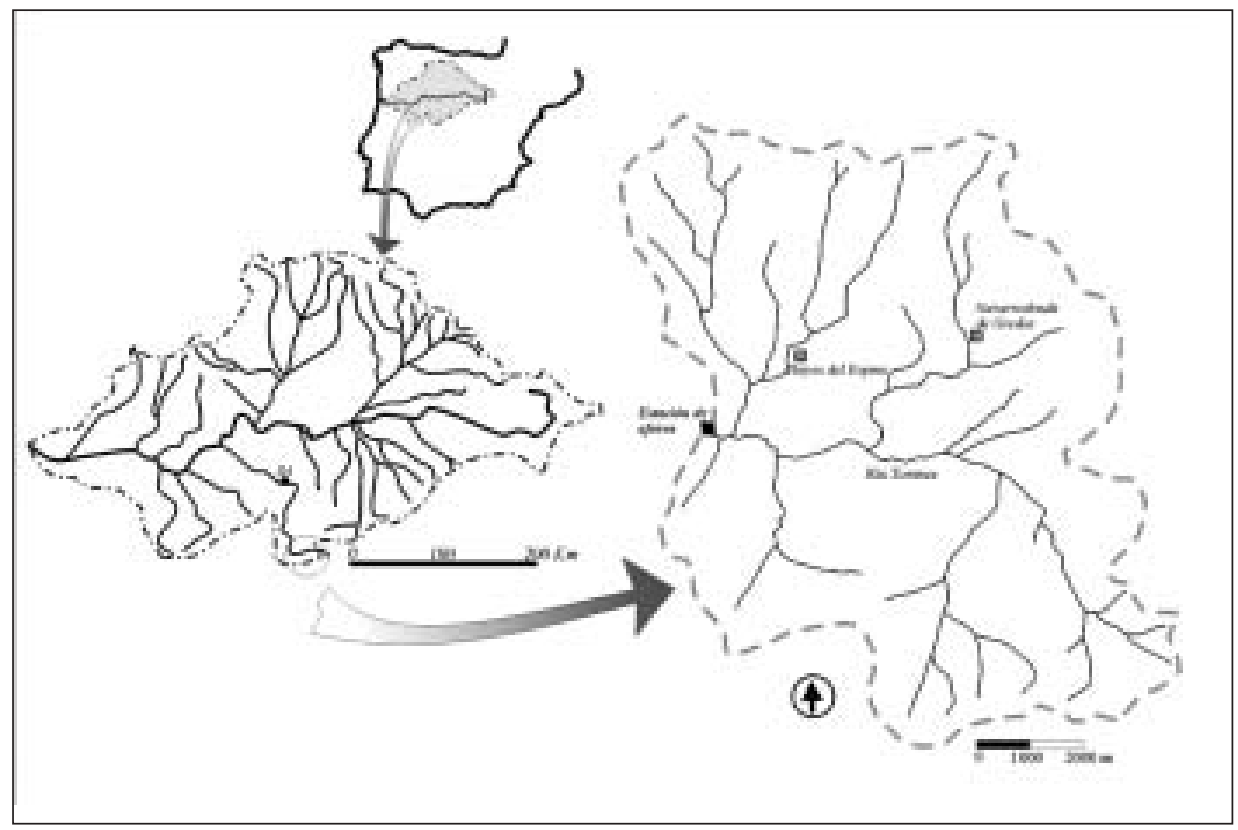

Figura 1. Localización de la zona de estudio y mapa con la red de drenaje y situación de la estaciones de aforos y pluviométrica

La litología predominante corresponde a rocas plutónicas del Hercínico (granitoides biotíticos, leucogranitos y migmatitas) y en los fondos de valle hay algunos sedimentos aluviales del Cuaternario (gravas, arenas, limos y arcillas).

En la vertiente norte del Sistema Central, la precipitación anual promedio de las estaciones meteorológicas de Navarredonda de Gredos, Hoyos del Espino y Bohoyo (todas por encima de los $1100 \mathrm{~m}$ de altitud) oscila en torno a los $900 \mathrm{~mm}$, con dos meses (julio y agosto) con precipitaciones medias inferiores a $25 \mathrm{~mm}$. La temperatura anual media oscila entre 10 y $10,5^{\circ} \mathrm{C}$, siendo enero el mes más frío con $3^{\circ} \mathrm{C}$ y no superándose los $20^{\circ} \mathrm{C}$ en el mes más cálido. De forma general, podemos catalogar el clima de la zona como mediterráneo con un claro carácter continental favorecido por la altitud.

Las dos terceras partes de la cuenca pertenecen al denominado Espacio Natural Protegido de la Sierra de Gredos, con una clara vocación forestal (Azcárate y García, 1992). Por debajo de los $1500 \mathrm{~m}$ son significativas las manchas de pino albar (Pinus sylvestris) y matorral de leguminosas (Cytisus multiflorus, C. scoparius, Genista cinerea, G. florida, G. scorpius, Adenocarpus sp., etc), así como algunas pequeñas parcelas de pastos y frutales (Foto 1). Por encima de los $1500 \mathrm{~m}$ abundan los piornales (Cytisus oromediterraneus y Echinospartum bardanessi) y pastizales de montaña (Festuca indigesta, Nardus stricta). Históricamente la agricultura ha tenido pocas posibilidades de desarrollo debido al rigor climático y a la pobreza de los suelos, siendo la explotación forestal, liga- 
da a la carretería, y la ganadería, muy vinculada a la disponibilidad de pastos montanos estivales, actividades de cierta importancia en la zona (Barrientos Alfageme, 1978).

En este territorio la densidad poblacional es baja, con dos entidades municipales (Hoyos del Espino y Navarredonda de Gredos) que en la actualidad suman algo menos de 1000 habitantes.

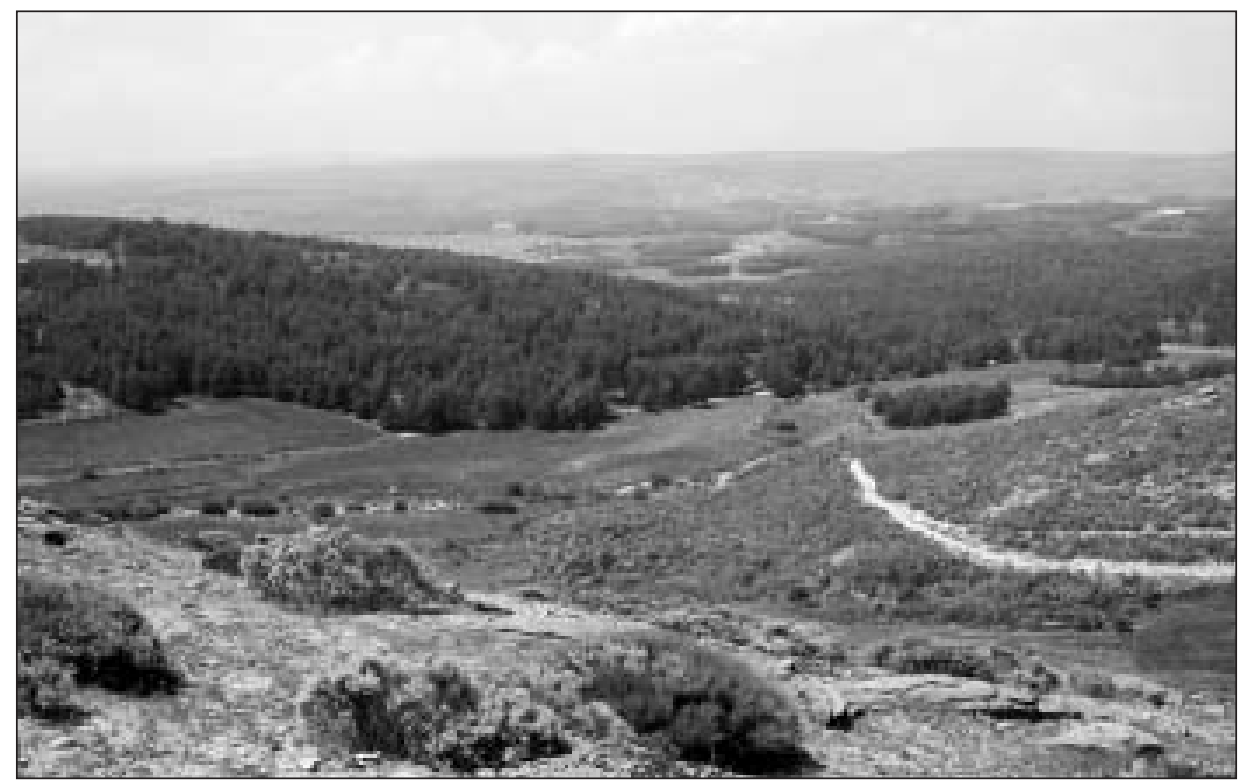

Foto 1. Vista general de la cabecera fluvial del río Tormes

\section{Métodos y técnicas}

Para el análisis de las temperaturas, la serie de datos corresponde al observatorio meteorológico de la ciudad de Ávila (40³9’ N; 4³9’ O; $1.126 \mathrm{~m}$ ), ya que se trata de la estación más próxima a la zona de estudio con una serie de datos continua y significativa en cuanto a su duración (1961-2005), y con una posición geográfica comparable. El estudio de las anomalías de las temperaturas ha consistido en calcular la diferencia en ${ }^{\circ} \mathrm{C}$ entre la temperatura media correspondiente a cada año, estación o mes, y la media de un período normal de referencia de 30 años (en nuestro caso hemos seleccionado el ultimo período climático normal, 1961-90). El resultado se ha expresado gráficamente observando la tendencia mediante la representación de la media móvil correspondiente a un período de 5 años (IPCC, 2001). Para contrastar el grado de significación de la tendencia (positiva, negativa o nula), con un determinado nivel de confianza, se aplicará un test no paramétrico como la correlación de rangos de Spearman (Rs) según recomiendan varios autores (Sneyers, 1992; Yue et al., 2002). 
Para analizar la tendencia de las precipitaciones se ha utilizado una serie de más de 50 años (1951-2002) correspondiente a la estación pluviométrica de Hoyos del Espino $\left(40^{\circ} 21^{\prime} \mathrm{N} ; 5^{\circ} 10^{\prime} \mathrm{O} ; 1.460 \mathrm{~m}\right)$, que se encuentra situada dentro del perímetro de la cuenca alta del Tormes. En primer lugar, se ha observado la dispersión de la muestra analizando la frecuencia de años secos y húmedos según la propuesta de Scian y Donnari (1997), para quienes año seco es aquel con valores inferiores a la precipitación media anual menos la desviación estándar y año húmedo es aquel con registros superiores a la precipitación media más la desviación estándar. Para definir el grado de variabilidad intraanual de la precipitación, así como su evolución temporal a lo largo del período de estudio (variabilidad interanual), se utilizará una versión modificada del denominado índice de concentración de lluvia (Precipitation Concentration Index, PCI), según la propuesta de Oliver (Oliver, 1980; De Luis et al., 2001).

$$
P C I=100 * \frac{\sum_{i=1}^{12} P_{i}^{2}}{\left(\sum_{i=1}^{12} P_{i}\right)^{2}}
$$

donde $p i$ es la cantidad de precipitación de un determinado número de meses $i$. Valores inferiores a 10 indican una distribución uniforme de la precipitación mensual a lo largo del año, valores comprendidos entre 11 y 20 denotan una cierta estacionalidad, y valores superiores a 20 son indicadores de una apreciable variabilidad en el reparto mensual de las precipitaciones anuales. Como en el caso de las temperaturas, se procederá a un análisis agrupando los datos en conjuntos estacionales y el significado de su tendencia será evaluado con un test no paramétrico.

En relación con el análisis hidrológico, se ha estudiado la evolución temporal de las aportaciones mensuales y anuales $\left(\mathrm{Hm}^{3}\right)$ medidas en la estación de aforos de Hoyos del Espino entre los años hidrológicos 1940-41 y 2003-04, aplicando los citados tests estadísticos para valorar las tendencias de las series.

Con el objetivo de comparar la evolución temporal de precipitaciones y temperaturas con la observada en las aportaciones hídricas, este conjunto de variables se han normalizado aplicando el índice $(F)$ propuesto, entre otros, por Shorthouse y Arnell (1999) y Beguería et al. (2003). Se trataría de normalizar las series anuales originales de datos restando a cada valor anual la media del conjunto del período de observación $(M d)$ y dividiendo el resultado por la desviación estándar de la serie $(S D)$. El resultado de esta operación es un índice sintético cuyo valor 0 corresponderá a aquellos años con un registro similar a $M d$ y el valor \pm 1 corresponderá a aquellos años con un registro igual a $M d$ $\pm \mathrm{SD}$. Por medio de la representación gráfica de las series temporales, una vez normalizadas, se podrá ver el paralelismo, o no, entre el comportamiento de las variables climáticas y el de las propias aportaciones a través del tiempo. Una notable ventaja de este índice es que ante la imposibilidad de un balance de agua para la cuenca, debido a que las entradas reales son superiores a las medidas en la única estación pluviométrica disponible, la normalización de las series permite una más fácil comparación entre varia- 
bles al utilizar la misma escala y posibilita analizar los cambios en las relaciones entre variables a lo largo del tiempo. Este análisis se ha realizado para el período comprendido entre los años hidrológicos 1961-62 y 2001-02, debido a la coincidencia temporal de las series de temperaturas, precipitaciones y aportaciones.

Finalmente los cambios experimentados en la cobertura forestal de la cuenca han sido estimados a través de la fotointerpretación del vuelo americano de 1957 y el análisis de las ortofotos correspondientes al vuelo de la Junta de Castilla y León durante los años 2001 y 2002. Este análisis ha sido completado con la información publicada en los tres inventarios forestales nacionales (Ministerio de Agricultura, 1975; Ministerio de Agricultura, Pesca y Alimentación, 1994 Junta de Castilla y León, 2005), el mapa de vegetación del espacio natural protegido de la Sierra de Gredos (Azcárate y García, 1992) y las propias observaciones de campo.

\section{Resultados y discusión}

\subsection{Variables climáticas: temperaturas y precipitaciones}

La temperatura media anual del observatorio de Ávila correspondiente al conjunto del período $1961-2005$ ha sido de $10,6^{\circ} \mathrm{C}$, siendo enero el mes más frío $\left(3,1^{\circ} \mathrm{C}\right)$ y julio el más cálido $\left(20,1^{\circ} \mathrm{C}\right)$. El análisis de las anomalías térmicas (Fig. 2) muestra dos períodos muy contrastados en cuanto a la tendencia de la serie: desde el año 1961 hasta 1972 se aprecia un notable descenso de la temperatura media anual respecto al período de referencia (1961-1990), mientras que entre 1973 y 2005 la tendencia al calentamiento es clara $\left(0,06^{\circ} \mathrm{C}\right.$ año $\left.{ }^{-1}\right)$, siendo idéntica a la observada por De Castro et al. (2005) en el conjunto del territorio español $\left(0,6^{\circ} \mathrm{C} /\right.$ década). Considerando la serie en su totalidad ( $\mathrm{n}$ $=45)$ no hay una tendencia estadística significativa $(\mathrm{Rs}=0,35$; valor-p $=0,018)$, que sin embargo sí es muy apreciable para los últimos 33 años de la serie (1973-2005), con un coeficiente de correlación de Spearman (Rs) de 0,77 y un valor-p menor a 0,001 . No obstante, al análisis de la tendencia temporal de las anomalías térmicas estacionales muestra que esta tendencia positiva se debe al comportamiento de las estaciones primaveral y estival, ya que tanto en otoño como en invierno no se observa ningún tipo de tendencia (Fig. 3). La tendencia observada, con una clara inflexión en la década de los 70, responde al patrón general de comportamiento estudiado por De Castro et al. (2005) para el conjunto del territorio español, aunque a esta escala el calentamiento haya sido más evidente en los meses invernales. Sin embargo, nuestros resultados sí coinciden con las conclusiones del análisis realizado por Morales et al. (2005) para el período 1945-96 en Castilla y León, que no encuentran una tendencia significativa en las anomalías anuales y que también aprecian un comportamiento diferente para el subperiodo 1972-1996, con un incremento especialmente significativo en la primavera y el verano.

En relación con el comportamiento hidrológico de la cuenca alta del Tormes, el calentamiento observado en primavera y verano puede repercutir en una mayor demanda evapotranspirativa durante el período de actividad vegetal y en una más temprana fusión de la nieve caída durante el invierno. 


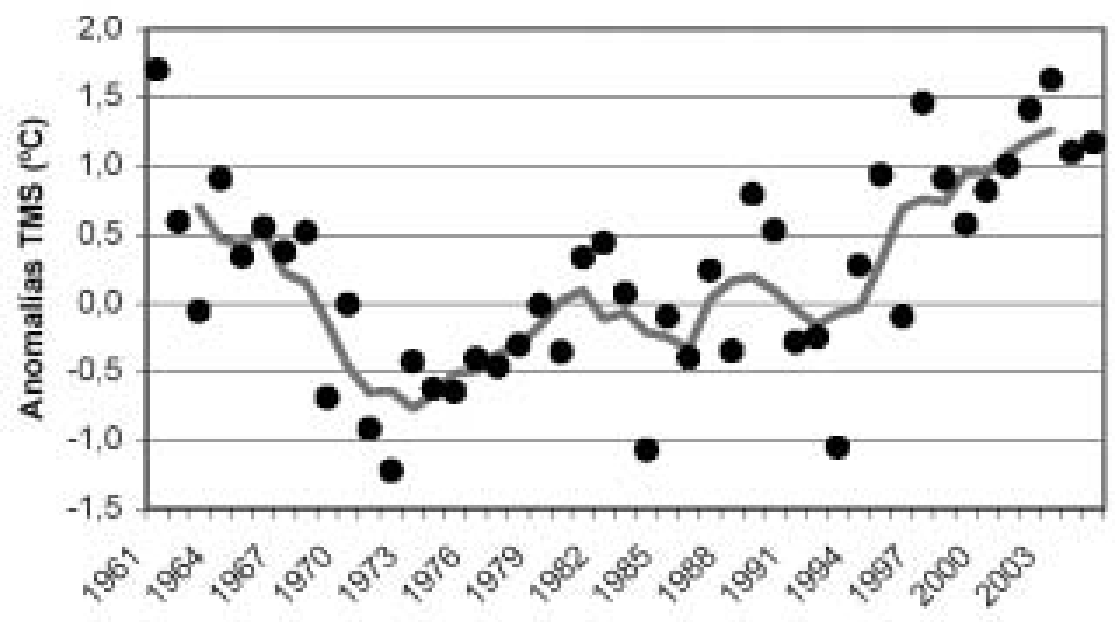

Años

Figura 2. Anomalías térmicas anuales respecto al período de referencia 1961-1990 (media móvil de 5 años). Observatorio Meteorológico de Ávila, 1961-2005

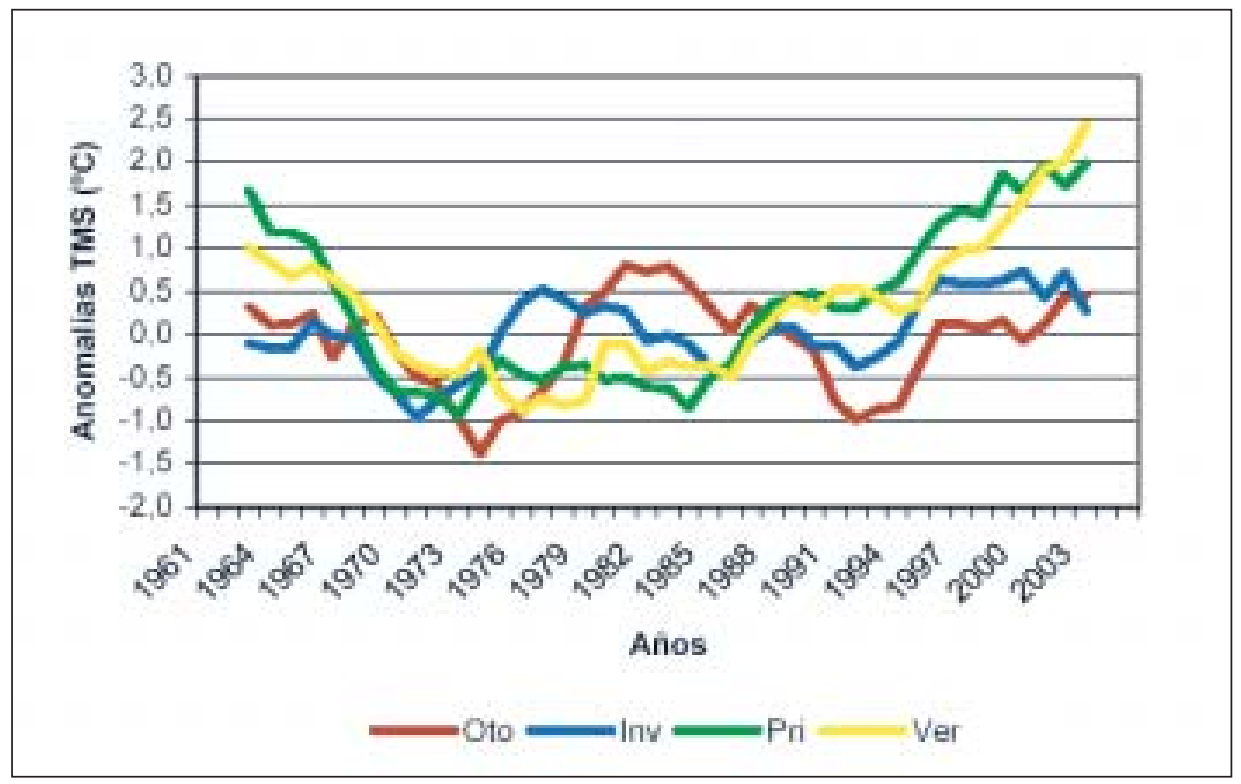

Figura 3. Anomalías térmicas estacionales respecto al período de referencia 1961-1990 (media móvil de 5 años). Observatorio Meteorológico de Ávila, 1961-2005 
La precipitación media anual para el conjunto de la serie (1951-2002) de la estación de Hoyos del Espino es de $846 \mathrm{~mm}$, con una desviación estándar (SD) de $241 \mathrm{~mm}$ y un coeficiente de variación (CV) del 28,5\%. Aunque la tendencia de la serie es negativa, sin embargo no es significativa desde un punto de vista estadístico ( $\mathrm{Rs}=-0,27$; valor-p $=0,055$ ) debido a que la variabilidad interanual se superpone a la tendencia de las series, por lo que difícilmente éstas serán estadísticamente significativas. No obstante, si se analiza la línea de tendencia el decrecimiento observado en las 5 décadas de la serie puede cuantificarse en un $22 \%$. En el gráfico correspondiente a la Fig. 4 puede observarse que el comportamiento de las precipitaciones es diferente en cuanto a su variabilidad interanual si distinguimos entre los períodos 1951-1979 y 1980-2002. En el primer caso, las precipitaciones son más abundantes y regulares, con un valor promedio de $915 \mathrm{~mm}$, una SD igual a $180 \mathrm{~mm}$ y un CV inferior al 20\%. Sin embargo la media correspondiente al segundo período es notablemente inferior $(758 \mathrm{~mm})$, con una mayor frecuencia de años muy húmedos y años muy secos en el contexto de la zona estudiada, lo cual se traduce en una mayor variabilidad interanual $(\mathrm{SD}=281 \mathrm{~mm} ; \mathrm{CV}=37,1 \%)$. Aunque el análisis general correspondiente a España no permita vislumbrar una tendencia en la variabilidad temporal de las precipitaciones, el comportamiento observado en la serie de Hoyos del Espino se adecuaría al detectado en algunos espacios regionales. Por un lado, en su estudio sobre el comportamiento espacio-temporal de las precipitaciones en Castilla y León para el período 1945-96, Labajo y Piorno (2001) encuentran una tendencia decreciente de las precipitaciones anuales aunque sin ningún significado estadístico. Por otro, al igual que en nuestro caso, González-Hidalgo et al. (2001) han

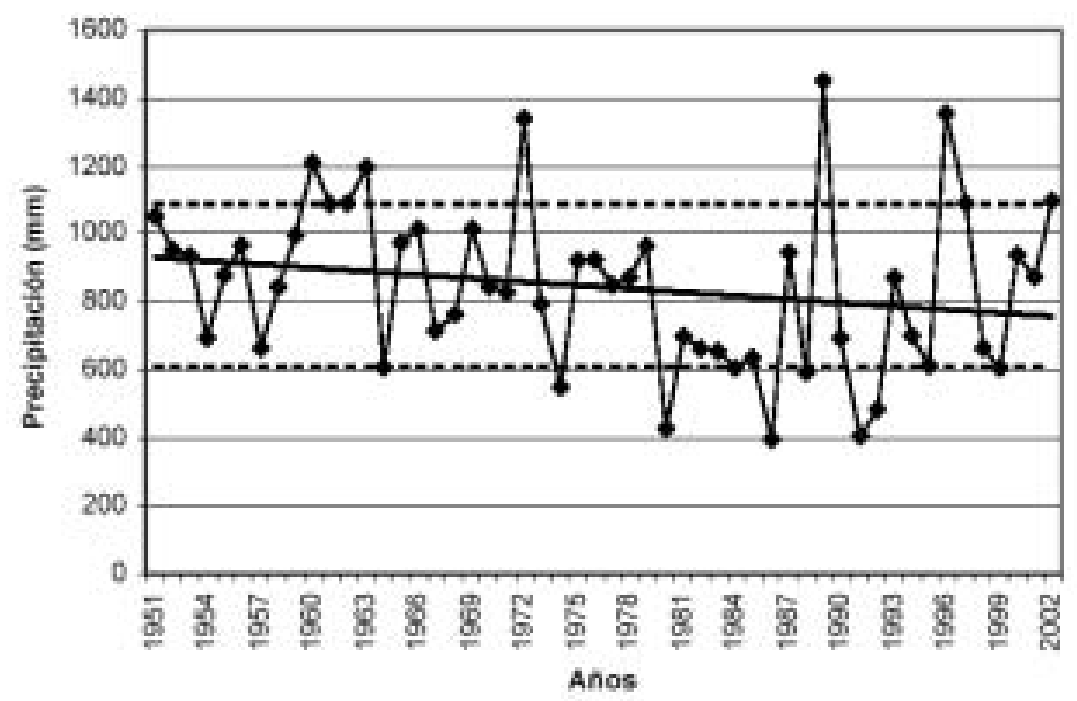

Figura 4. Evolución interanual de las precipitaciones en la estación pluviométrica de Hoyos del Espino, 1951-2002 (líneas discontinuas: Precipitación media \pm SD; $846 \pm 241 \mathrm{~mm}$ ) 
detectado un incremento de la variabilidad pluviométrica interanual en la Comunidad Valenciana. El análisis de las precipitaciones, agrupadas por períodos estacionales, ha mostrado siempre una tendencia negativa aunque en ningún caso significativa.

En relación con la evolución interanual de la distribución intranual de las precipitaciones (PCI), la serie muestra una tendencia positiva (Fig. 5) aunque estadísticamente no significativa $(\mathrm{Rs}=0,24$; valor-p $=0,095)$.

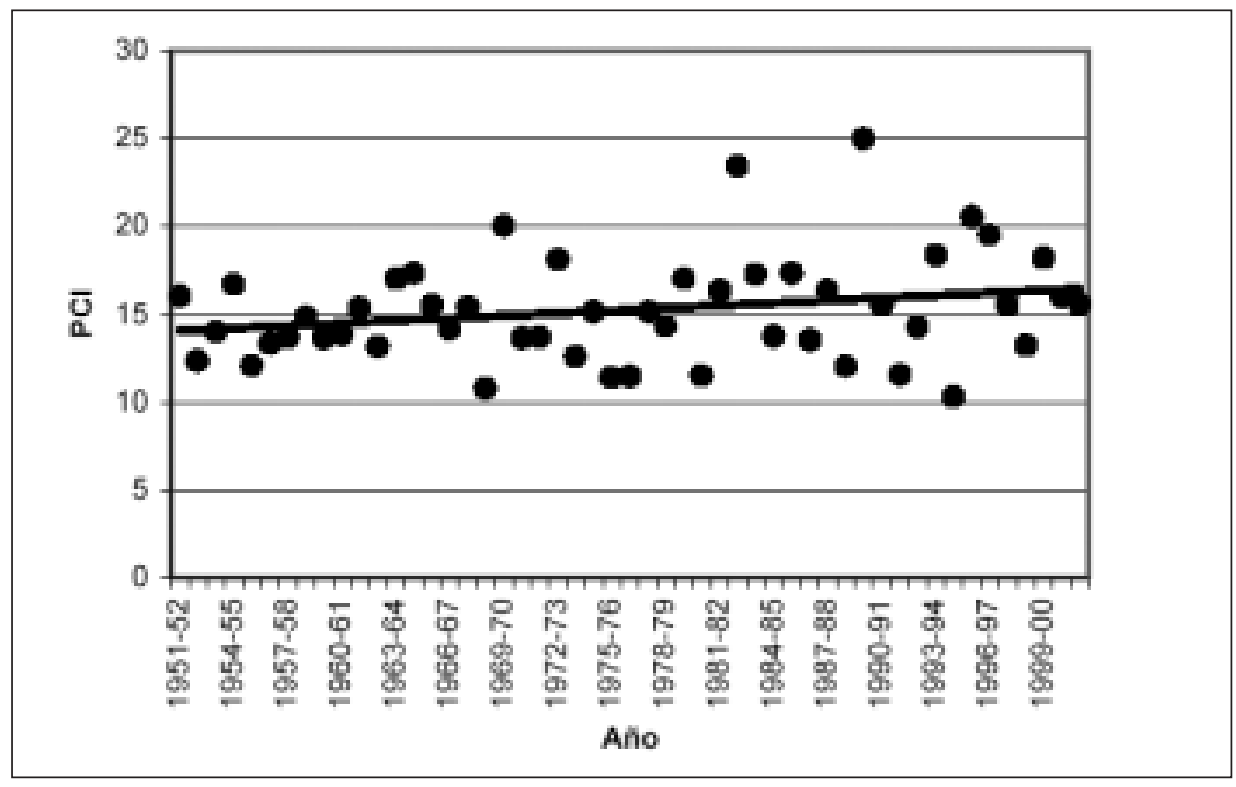

Figura 5. Evolución interanual del PCI en la estación pluviométrica de Hoyos del Espino, 1951-2002

\subsection{Aportaciones fluviales}

La evolución de las aportaciones anuales registrada en la estación de aforos de Hoyos del Espino para el período 1940-41/2003-2004 no muestra ninguna tendencia estadísticamente significativa $(\mathrm{Rs}=-0,07$; valor-p $=0,572)$, aunque como en el caso de las precipitaciones, a partir del análisis de la Fig. 6, la serie puede dividirse en dos subperíodos. Durante los 26 años comprendidos entre el comienzo de la serie y el año hidrológico 1965-66, la frecuencia de años con una aportación anual superior a los $80 \mathrm{Hm}^{3}$ es apreciable (11 años), mientras que en los 38 años restantes esta circunstancia se produjo sólo en una ocasión. La aportación media del primer subperiodo es superior a la del segundo (65,3 y $52,8 \mathrm{Hm}^{3}$ respectivamente), al igual que la variación interanual ( $\mathrm{CV}=$ $55,7 \%$ en el primer subperiodo y $34,9 \%$ en el segundo). 


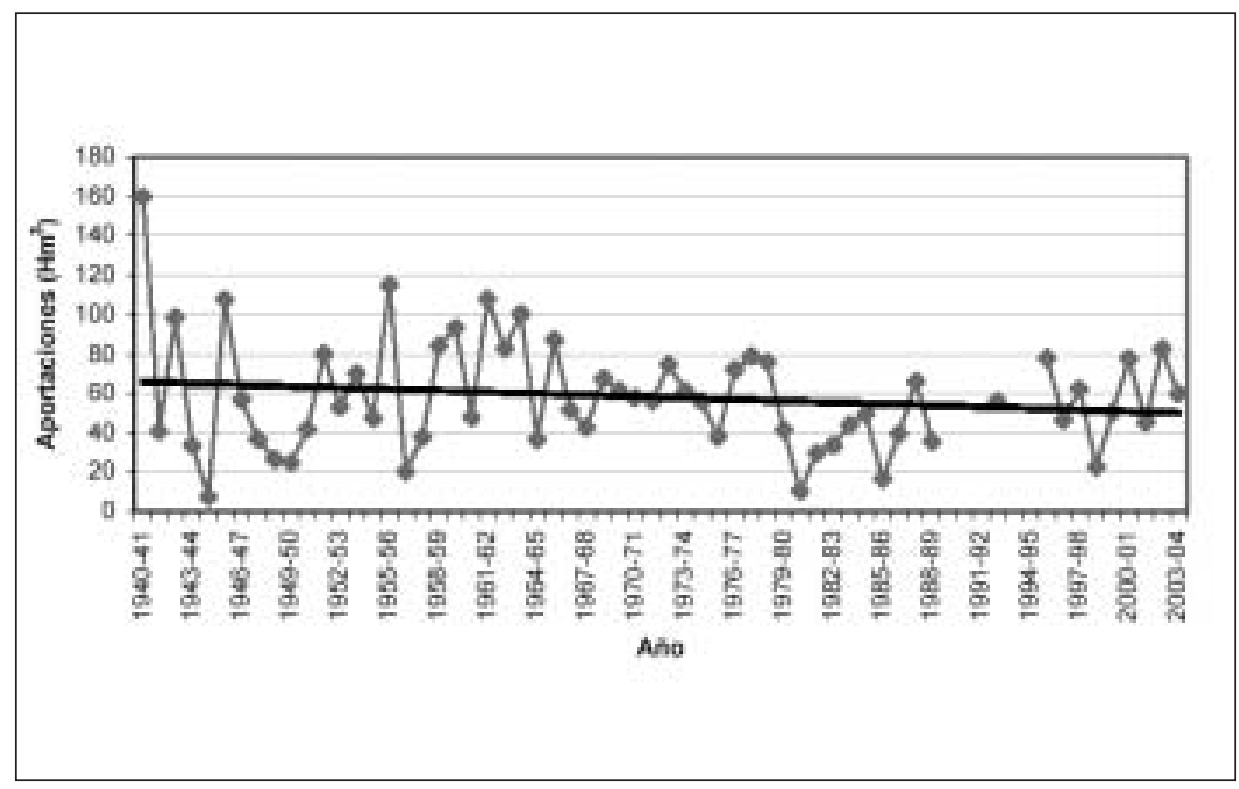

Figura 6. Evolución interanual de las aportaciones en la estación de aforos de Hoyos del Espino, 1940- 41/2003-04

Considerando la serie de aforos completa, la aportación media ha sido de 58,3 \pm 28,2 $\mathrm{Hm}^{3}$, con un $\mathrm{CV}=48,4 \%$. La distribución media mensual de las aportaciones (tabla $1 \mathrm{y}$ Fig. 7) es la típica de un régimen nivo-pluvial, como ya describieron algunos autores que estudiaron las primeras décadas de la serie de datos (Masachs Alavedra, 1948; Barrientos Alfageme, 1978), mostrando dos máximos, uno principal en abril de carácter nival y un segundo en diciembre de carácter pluvial. Entre 1951 y 2002 coinciden las series de precipitación y aportación, lo cual nos permite analizar si la distribución mensual de las aportaciones ha cambiado a lo largo de la segunda mitad del siglo XX o por si, al contrario, ese régimen nivo-pluvial que caracteriza al conjunto de la serie ha sido una constante en

Tabla 1: Distribución mensual y anual de las aportaciones medias, desviación estándar (SD), valores máximo y mínimo, correspondientes a la serie de datos estudiada. Estación de aforos de Hoyos del Espino (1940-41/2003-2004). Datos expresados en $\mathrm{Hm}^{3}$

\begin{tabular}{|l|r|r|r|r|r|r|r|r|r|r|r|r|c|}
\hline & Oct & Nov & Dic & Ene & Feb & Mar & Abr & May & Jun & Jul & Ago & Sep & Año \\
\hline Media & 2,91 & 6,13 & 6,76 & 6,61 & 5,73 & 7,84 & 8,77 & 8,60 & 2,98 & 1,23 & 0,71 & 1,07 & $\mathbf{5 8 , 3}$ \\
SD & 3,55 & 5,87 & 6,59 & 5,66 & 4,30 & 5,58 & 5,54 & 10,04 & 1,90 & 1,01 & 0,89 & 1,10 & $\mathbf{2 8 , 2}$ \\
Máx. & 15,18 & 25,65 & 29,81 & 22,53 & 22,47 & 23,59 & 34,87 & 77,86 & 10,05 & 5,98 & 5,41 & 5,37 & $\mathbf{1 5 9 , 2 6}$ \\
Mín. & 0,17 & 0,24 & 0,52 & 0,58 & 0,97 & 0,57 & 2,09 & 0,76 & 0,44 & 0,24 & 0,13 & 0,10 & $\mathbf{7 , 9 4}$ \\
\hline
\end{tabular}




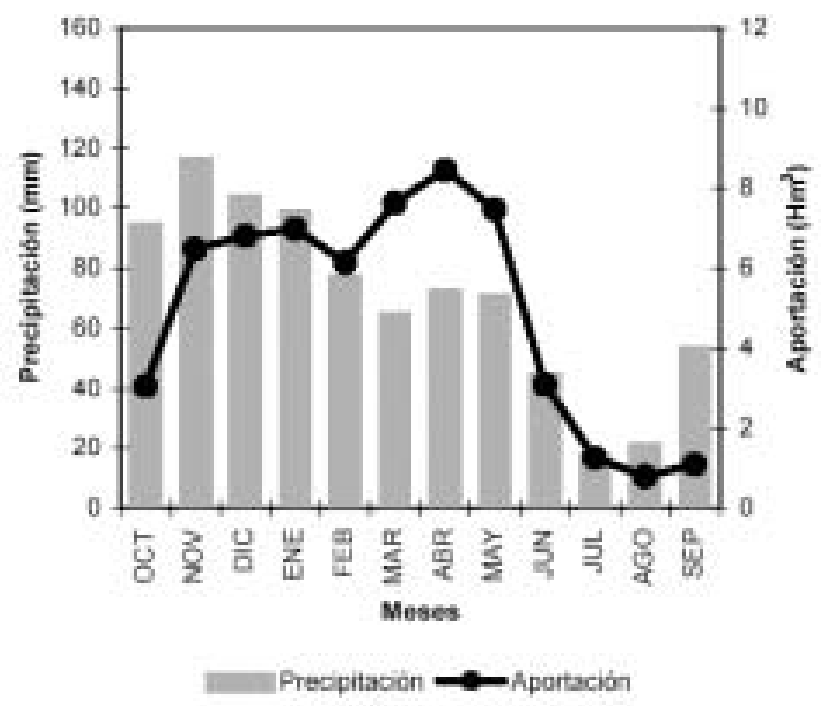

Figura 7. Distribución mensual de las aportaciones y precipitaciones en el curso alto del río Tormes (1951-52/2001-2002)

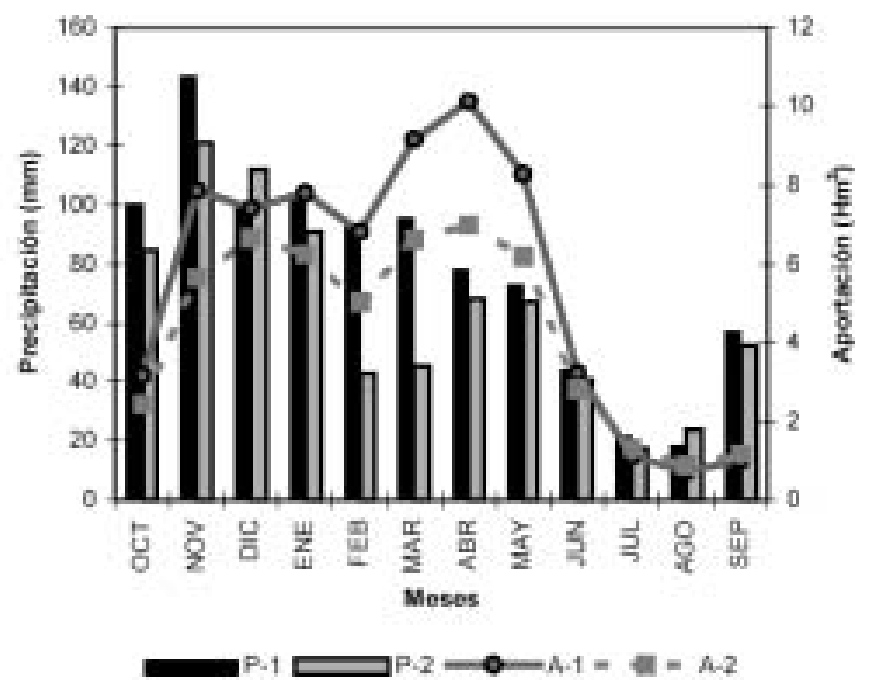

Figura 8. Comparación entre la distribución mensual de las aportaciones (A) y precipitaciones $(P)$ en el curso alto del río Tormes en los períodos comprendidos entre 1951-52/1971-72 (1) y 1981-82/2001-02 (2) 
el comportamiento del río. La comparación entre la media correspondiente a las dos primeras décadas de la serie común y las dos últimas (Fig. 8) sugiere un cambio importante en el régimen del río, que iría perdiendo su carácter nival al igualarse la magnitud de los dos máximos mensuales en las aportaciones. Las menores precipitaciones en los meses de febrero y marzo, junto al incremento térmico apreciable en las series de temperatura, pueden explicar la evidente disminución de las aportaciones en el mes de abril.

\subsection{Cambios en el bosque}

El aumento de la superficie forestal en España en las últimas cuatro décadas es un hecho que actualmente nadie cuestiona (Ministerio de Medio Ambiente, 2000). Por un lado, ha jugado un papel relevante el abandono de determinados espacios rurales y, por otro, ha sido un proceso favorecido por las políticas autonómicas, estatal y europea debido al efecto positivo de la cubierta forestal para la conservación del suelo, absorción de carbono, mantenimiento de la diversidad biológica y regulación del ciclo hidrológico (OSE, 2006).

Un estudio recientemente publicado por la Junta de Castilla y León, basado en los datos de las tres inventarios forestales nacionales, concluye que el monte arbolado ha aumentado su superficie cerca de un $60 \%$ en dicha comunidad en los últimos 40 años (Junta de Castilla y León, 2005). En el caso de la provincia de Ávila, los datos correspondientes a los tres inventarios forestales publicados hasta el momento revelan una clara progresión de la superficie arbolada. El primer inventario forestal muestra la situación de los bosques abulenses a finales de la década de los 60, con 117.300 ha (Ministerio de Agricultura, 1975). El segundo inventario forestal expresa un notable incremento a comienzos de los 90, con un balance de 158.131 ha (Ministerio de Agricultura, Pesca y Alimentación, 1994). Los datos del tercer inventario forestal reflejan la situación a comienzos de la presente década, con un sustancial crecimiento del $51 \%$ en tan sólo 10 años -239.307 ha- (Junta de Castilla y León, 2005). Por lo tanto, el balance global es que en algo menos de 40 años la superficie ocupada por los bosques en la provincia de Ávila ha crecido en un $104 \%$, ocupando en la actualidad un tercio de la superficie provincial.

Con el objetivo de comprobar si esta tendencia provincial es aplicable a la cuenca estudiada, hemos planimetrado las superficies arboladas a partir de las fotografías aéreas del vuelo de 1957 y ortoimágenes correspondientes a los años 2001 y 2002 (Fig. 9). A finales de la década de los 50 el bosque monoespecífico de Pinus sylvestris ocupaba un total de 982 ha, mientras que a comienzos del siglo XXI la cifra se eleva a 1.279 ha. Es decir, en algo más de 40 años, la superficie arbolada de la cabecera fluvial del Tormes ha crecido un 30\%. De acuerdo con la Fig. 9 y las observaciones de campo, el aumento se debe sustancialmente al incremento del número de pequeños rodales aislados de plantaciones forestales gestionadas por los servicios de selvicultura de la Junta de Castilla y León, ya que el perímetro del bosque histórico, en las proximidades del curso principal, no ha experimentado cambios importantes.

Aunque la población de los dos municipios de la zona haya decrecido en los últimos 40 años (1.504 habitantes en 1960 frente a 936 en 2000), así como la intensidad de algunas 


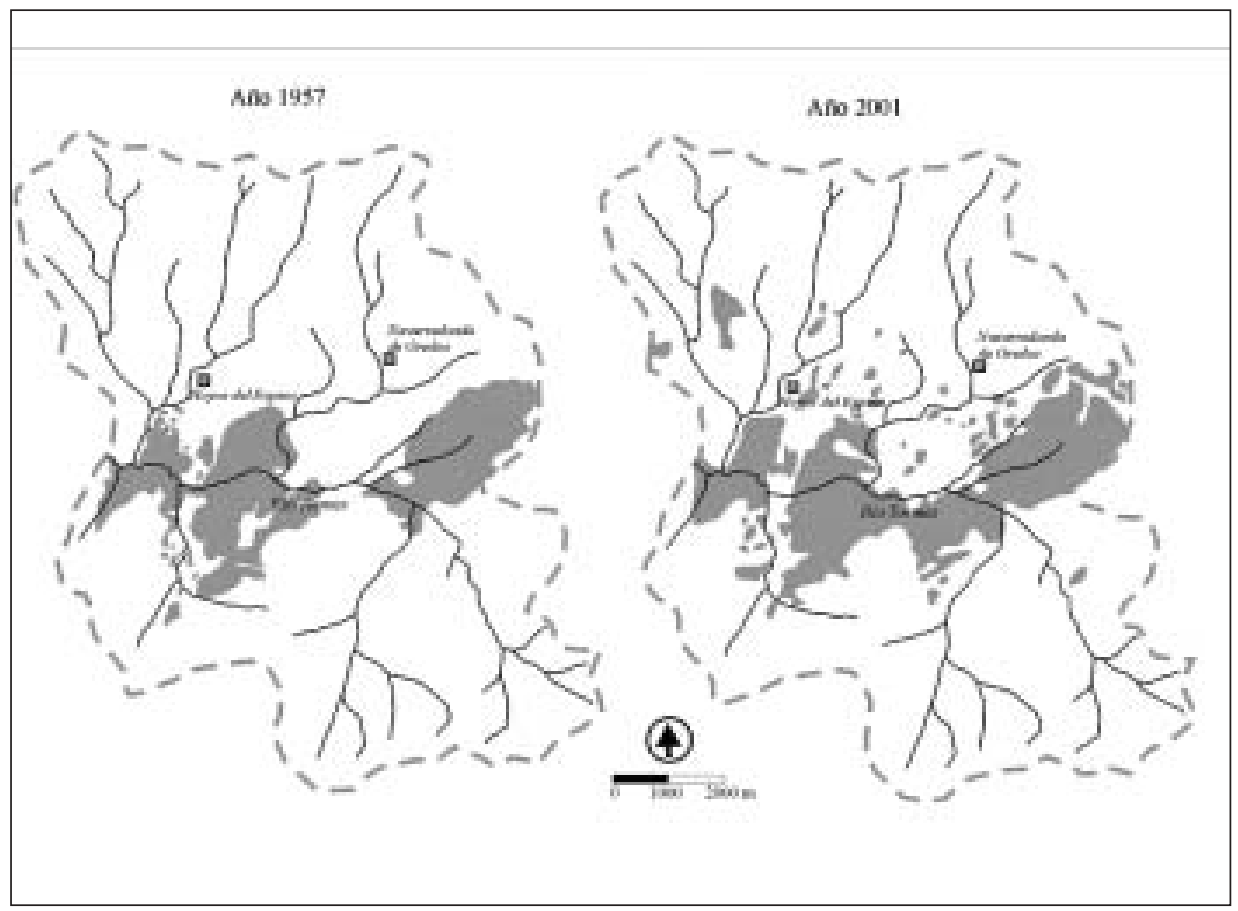

Figura 9. Distribución de la superficie forestal en 1957 y en 2001 en la cabecera fluvial del río Tormes

actividades como la agricultura, el avance del bosque en la cuenca analizada no se debe al abandono del campo como han apuntado algunos autores en referencia a otros ecosistemas de montaña en nuestro país (García Ruiz et al., 2001). Las observaciones de campo realizadas en la cabecera fluvial del Tormes han permitido comprobar un grado de intervención importante en las masas forestales por parte de la administración (labores de limpieza, podas, nuevas repoblaciones, cortafuegos, pistas de acceso, etc), lo cual nos permite afirmar que no se trata de un ecosistema abandonado en donde la recuperación natural de la vegetación sea evidente. Junto a ello, muchos prados de siega situados en las proximidades del río siguen siendo mantenidos por la población, lo cual hipoteca el avance del bosque en un área favorable para ello. Esta vocación forestal del territorio ha sido secular, con un alto grado de implicación de la población, y siempre dependiente de administraciones públicas como ayuntamientos, Patrimonio Forestal del Estado, etc (Barrientos Alfageme, 1978).

Por lo tanto, la única opción que tiene el bosque para crecer de forma natural es hacerlo hacia la línea de cumbres, ocupando el área actual del piorno (Cytisus oromediterraneus). No obstante, la mala calidad del suelo, con afloramientos superficiales del roquedo granítico, actúa como un factor físico limitante para una especie como Pinus sylvestris, que necesita suelos profundos y frescos (Ruiz de la Torre y Ceballos, 1979) para aguantar el estrés hídrico estival propio de la montaña mediterránea. 


\subsection{Interacciones entre las variables hidroclimáticas y el posible efecto del bosque}

La Fig. 10 refleja la evolución temporal de las temperaturas, precipitaciones y aportaciones anuales una vez normalizadas. Es destacable que la trayectoria de las líneas correspondientes a las precipitaciones y aportaciones sea muy parecida, solapándose en varias ocasiones, lo que sugiere un grado de correlación apreciable entre ambas variables. El coeficiente de determinación (Fig. 11) entre precipitación y aportación es significativo $\left(\mathrm{r}^{2}=0,708\right.$; valor-p $\left.<0,001\right)$. A diferencia de otros análisis, como el de Beguería et al. (2003), la inclusión de variables como la temperatura o el tiempo en un análisis de regresión paso a paso no mejora el grado de explicación de la variable dependiente.

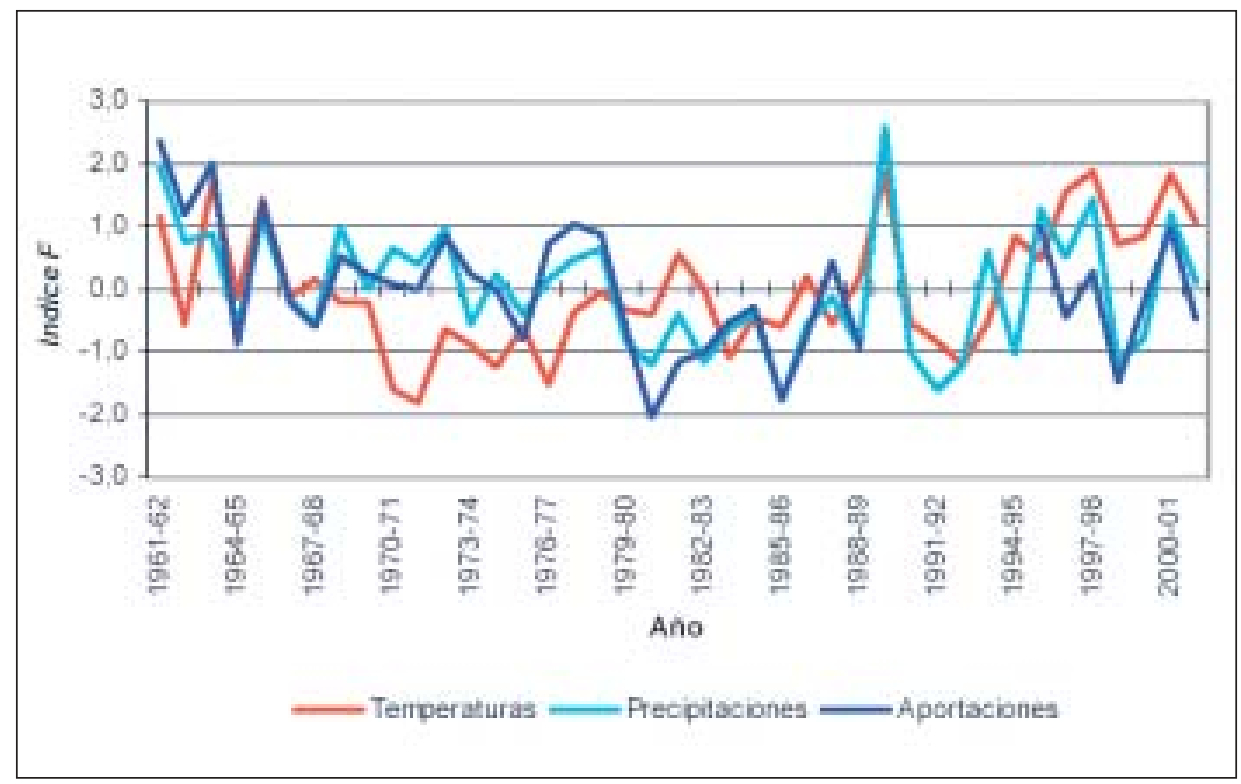

Figura 10. Evolución temporal de las temperaturas, precipitaciones y aportaciones normalizadas (1961-62/2001-2002)

Los mencionados autores, en su estudio de las relaciones entre caudales de los ríos, oscilaciones climáticas y cambios en el uso del suelo en los Pirineos, destacaron que una señal evidente del efecto del bosque sobre los caudales es que la relación entre precipitación y aportación cambie a lo largo del período de estudio. Una de las más claras y evidentes consecuencias hidrológicas de la extensión del bosque es que la producción escorrentía decrezca para una determinada cantidad de lluvia, lo cual sería un claro indicador de un mayor consumo de agua por evapotranspiración. En el caso del tramo de la cuenca estudiada, no hay evidencias de que ello ocurra, ya que no hay ningún subperíodo en el que se observe que sistemáticamente el valor del índice de normalización correspondiente a las aportaciones esté por debajo del de las precipitaciones. Aunque en 


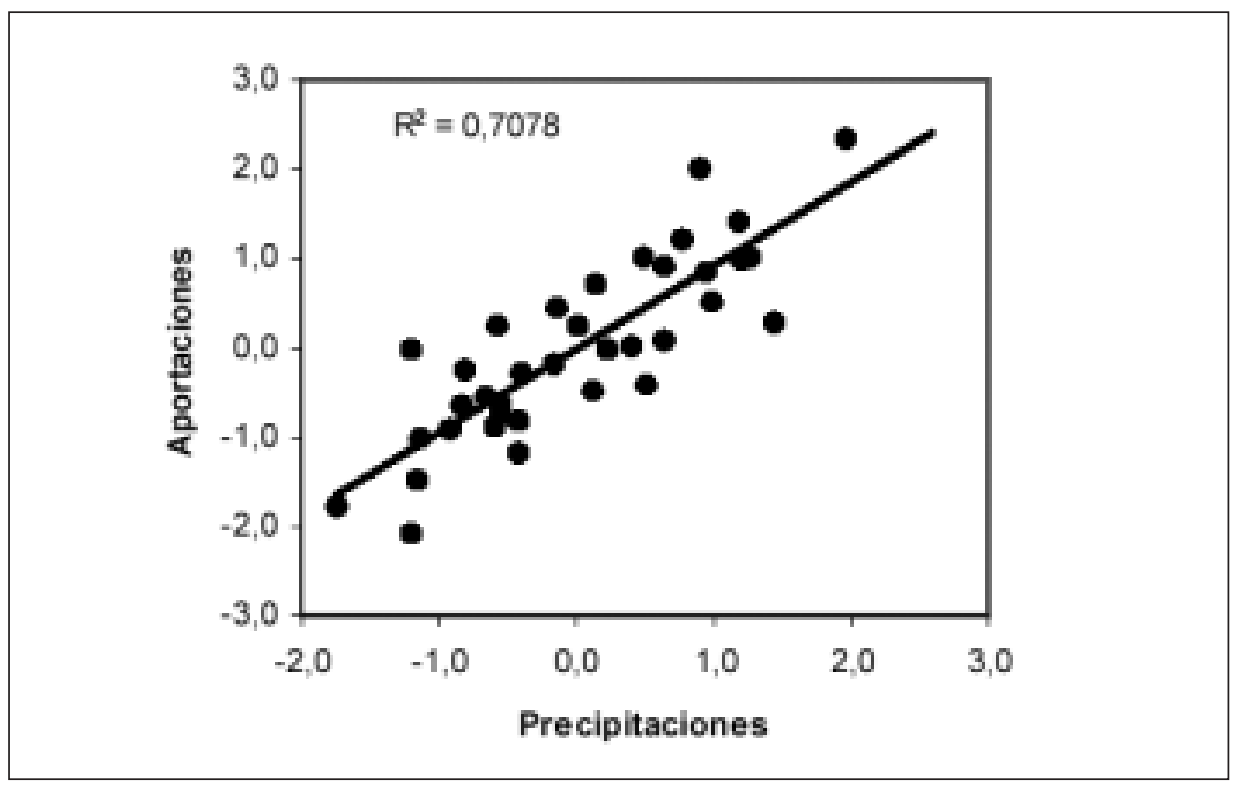

Figura 11. Análisis de regresión entre las precipitaciones y aportaciones anuales normalizadas. Hoyos del Espino (1961-62/2001-02)

el área estudiada la superficie arbolada haya aumentado en un 30\% en 40 años, es posible que su influencia sea limitada sobre la producción de escorrentía en la cuenca debido a la discontinuidad espacial de las nuevas manchas boscosas. Incluso cabe argumentar que el posible decrecimiento de la escorrentía, debido a los nuevos cultivos forestales, haya sido compensado con el aumento de escorrentía causado por la apertura de pistas de acceso a las parcelas forestales (Úbeda et al., 1998; Arnáez et al., 2004).

Aunque en apartados previos hemos analizado las tendencias observadas y su significado estadístico de las series de datos anuales, la Fig. 12 refleja los coeficientes de correlación de Spearman correspondientes a las series mensuales del período en el que coinciden los datos de temperaturas, precipitaciones y aportaciones.

Al igual que en el caso de las series anuales, no hay una tendencia clara, y sobre todo estadísticamente significativa (nivel de confianza p <0,05). En el caso de las temperaturas, como ya se señaló previamente, hay un aumento general, más evidente en los meses primaverales y estivales, aunque sólo en marzo el incremento es significativo. Las precipitaciones, salvo en mayo y agosto, decrecen en todos los meses, siendo este descenso significativo en febrero y en marzo como también han observado García Ruiz et al. (2001) en el Pirineo aragonés.

Finalmente, en el caso de las aportaciones, las series mensuales muestran un comportamiento más variable en cuanto a sus tendencias ya que entre junio y octubre hay una tendencia al incremento de las aportaciones, aunque en ningún caso significativa, 


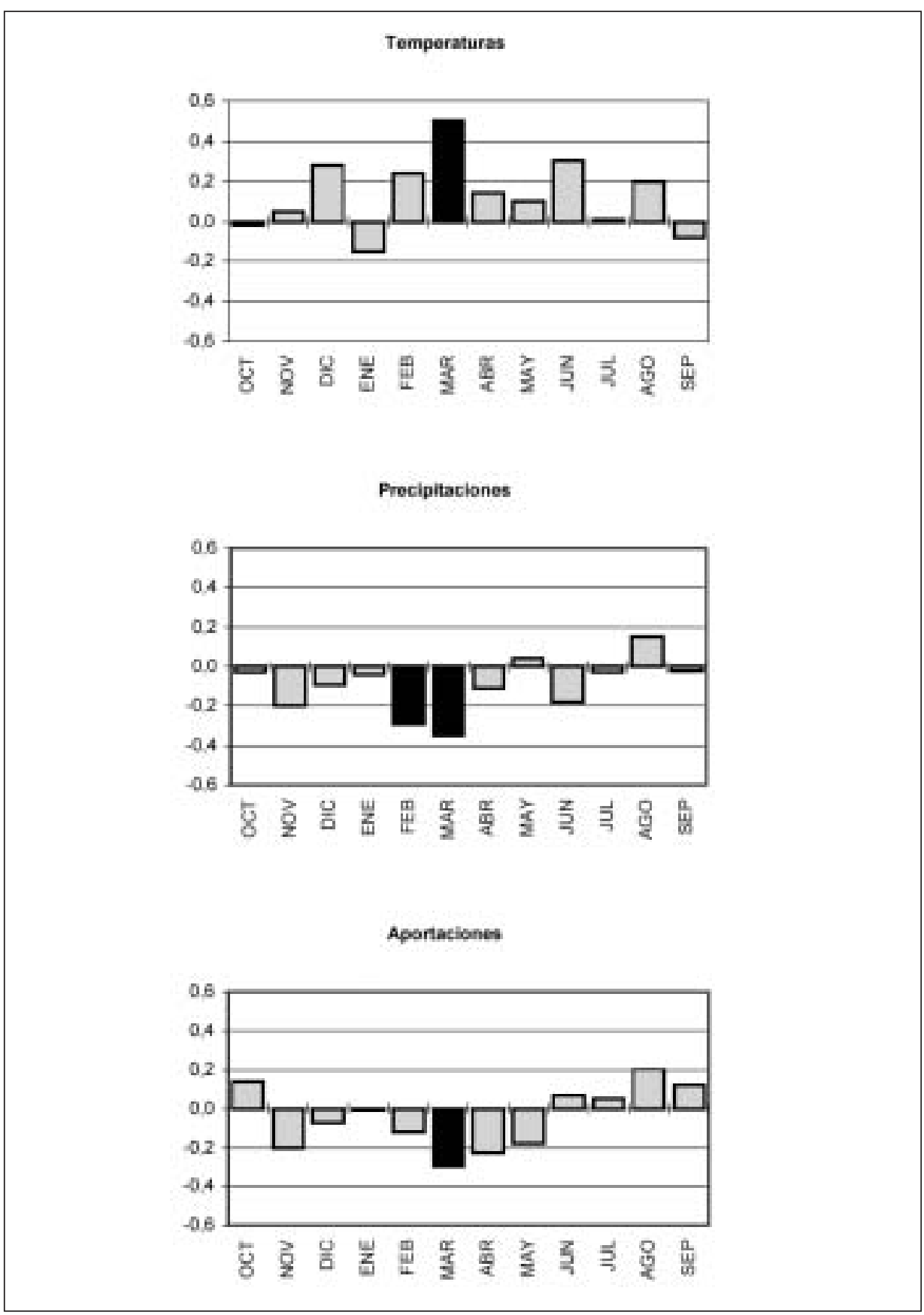

Figura 12. Análisis de las series mensuales de temperatura, precipitación y aportaciones (período 1961-62/2001-02) mediante la correlación de rangos de Spearman. Las correlaciones significativas a un nivel de confianza $p<0,05$ muestran una trama negra 
mientras que entre noviembre y mayo todos los meses muestran una tendencia negativa, aunque sólo en el caso de marzo sea estadísticamente significativa. Desde la perspectiva de los aportes de la cuenca alta del Tormes al resto del área subsidiaria, este incremento es imperceptible ya que afecta a los meses en los que los caudales son muy bajos y el río sufre un claro estiaje.

A partir de estos datos se puede parcialmente explicar el cambio de régimen fluvial comentado en el apartado 4.2 (Fig. 8), ya que las menores precipitaciones en los meses de febrero y marzo (en forma de nieve en las partes más altas de la cuenca), junto al incremento de las temperaturas, muy significativo en marzo, hayan repercutido en una suavización del pico de abril en la curva de las aportaciones. Este comportamiento ha sido detectado en el Pirineo Aragonés por García-Ruiz et al. (2001), quienes señalan una regresión de las aportaciones en los meses primaverales como consecuencia de una menor retención de nieve y un menor peso de las aguas de fusión.

\section{Conclusiones}

Los resultados del presente estudio confirman un aumento sostenido de las temperaturas a partir de la década de los setenta a un ritmo de $0,06^{\circ} \mathrm{C}$ año ${ }^{-1}$. Las precipitaciones han mostrado una tendencia negativa a lo largo del período de estudio, con un aumento de la variabilidad interanual.

Las aportaciones fluviales muestran un decrecimiento a través del tiempo, aunque estadísticamente no significativo, resultando de gran interés el cambio detectado en la distribución media mensual de las mismas. La disminución de las precipitaciones en los meses de febrero y marzo, y el notable aumento de las temperaturas en el mes de marzo han provocado que el régimen fluvial de la cuenca haya perdido su carácter nival en las últimas décadas. Esta disminución de los aportes primaverales puede repercutir negativamente en la gestión de la totalidad de la cuenca del Tormes, ya que embalses como el de Santa Teresa dependen de ellos para el mantenimiento de las reservas durante la estación estival.

A pesar de que durante la segunda mitad del siglo XX la extensión de la superficie arbolada en la cabecera fluvial del Tormes ha aumentado en un $30 \%$, el análisis conjunto de las variables hidroclimáticas normalizadas muestra un nivel de correlación muy significativo entre precipitaciones y aportaciones anuales que ha permanecido constante a lo largo del tiempo y no se aprecian indicios de que este crecimiento del bosque haya repercutido en una disminución de las aportaciones fluviales.

Finalmente, el comportamiento observado en la cuenca estudiada, representativa de los ambientes montañosos mediterráneos del Sistema Central, es comparable al observado en otros sistemas montañosos como el pirenaico (García Ruiz et al., 2001), lo cual permite esbozar un patrón generalizable para gran parte del territorio peninsular. Los resultados que se publiquen en los próximos años permitirán confirmar con más o menos matices esta impresión general. 


\section{Agradecimientos}

Esta investigación ha sido posible gracias al apoyo financiero de la Junta de Castilla y León (Proyecto SA099A06). Los autores del trabajo agradecemos al Dr. Francesc Gallart la lectura crítica del trabajo y los comentarios y sugerencias derivados de la misma.

\section{Referencias bibliográficas}

ANDRÉASSIAN, V. (2004). Waters and forests: from historical controversy to scientific debate. Journal of Hydrology, 291: 1-27.

ARNÁEZ, J., LARREA, V. y ORTIGOSA, L. (2004). Surface runoff and soil erosion on unpaved forest roads from rainfall simulation tests in northeastern Spain. Catena, 57: 1-14.

ARnEll, N. (1998). Climate change and water resources in Britain. Climate Change, 39: 83-110.

AYAlA-CARCEDO, F.J. y IGLESIAS, A. (2000). Impactos del posible cambio climático sobre los recursos hídricos, el diseño y la planificación hidrológica en la España peninsular. El Campo de las Ciencias y las Artes, 137: 201-222.

AZCÁRATE LuXÁN, J.M. y GARCÍA ARRIBAS, M.P. (1992). Mapa de vegetación del Espacio Natural Protegido de la Sierra de Gredos. Monografías de la Red de Espacios Naturales de Castilla y León. Junta de Castilla y León, Valladolid.

Barrientos Alfageme, G. (1978). El valle alto del Tormes. Gredos y Aravalle: estudio geográfico. Caja Central de Ahorros y Préstamos de Ávila. 321 pp.

Beguería, S., López-Moreno, J.I., Lorente, A., Seeger, M. y García-Ruiz, J.M. (2003). Assessing the effect of climate oscillations and land-use changes on streamflow in the Central Spanish Pyrenees. Ambio, 32(4): 283-286.

De CAstro, M., Martín-Vide, J. y Alonso, S. (2005). El clima de España: pasado, presente y escenarios de clima para el siglo XXI. En: Moreno Rodríguez, J.M. (Coord.): Evaluación preliminar de los impactos en España por efecto del cambio climático. Ministerio de Medio Ambiente y Universidad de Castilla-La Mancha. pp. 1-64.

De Luis, M., García-CANO, M.F., Cortina J., RaVEntós, J., GOnZÁlez-HidAlgo, J.C. y SÁNCHEZ, J.R. (2001). Climatic trends, disturbances and short-term vegetation dynamics in a Mediterranean shrubland. Forest Ecology and Management, 147: 25-37.

DunNE, T. y LEOPOLD, L.B. (1978). Water in environmental planing. W.H. Freeman and Company, New York.

GALLART, F. y LlORENS, P. (2002). Water resources and environmental change in Spain. A key issue for sustainable catchment management. En: García Ruiz, J.M., Jones, 
J.A.A. y Arnáez, J. (Eds): Environmental Change and Water Sustainability. IPE-CSIC, Zaragoza. pp. 11-20.

GARCÍA-RUIZ, J.M. y GALLART, F. (1997). Las cuencas experimentales como base para el estudio de la erosión y la desertificación. En Ibáñez, J.J., Valero, B.L. y Machado, C. (eds): El paisaje mediterráneo a través del espacio y del tiempo. Implicaciones en la desertificación, pp. 221-238. Geoforma Ediciones, Logroño.

GARcíA-Ruiz, J.M., Beguería Portugués, S., LóPEZ Moreno, J.I., LORENTE GRIMA, A. y SEEgER, M. (2001). Los recursos hídricos superficiales del Pirineo aragonés y su evolución reciente. Geoforma Ediciomes, Logroño. 192 pp.

González Hidalgo, J.C., De Luis, M., Raventós, J. y SÁnchez, J.R. (2001). Spatial distribution of seasonal rainfall trends in a western Mediterranean area. International Journal of Climatology, 22: 1593-1615.

GuPTA, V. y WAYMIRE, E. (1990). Multiscaling properties of spatial rainfall and river flow distribution. Journal of Geophysical Research, 95(D3): 1999-2010.

Iglesias, A., Estrela, T. y Gallart, F. (2005). Impactos sobre los recursos hídricos. En Moreno Rodríguez, J.M. (Coord.): Evaluación preliminar de los impactos en España por efecto del cambio climático. Ministerio de Medio Ambiente y Universidad de Castilla-La Mancha. pp. 303-354.

IPCC (2001). Climatic Change 2001: The Scientific Bases. Contribution of Working Group I to the Tirad Assessment Report of the Intergovernmental Panel on Climate Change. Houghton, J.T., Ding, Y., Griggs, D.J., Noguer, M., van der Linden, P.J., Dai, X., Maskell, K. y Johnson, C.A. (eds). Cambridge University Press, United Kingdom. 881 pp.

JOFFRE, R. y RAMBAL, S. (1993). How tree cover influences the water balance of Mediterranean rangelands. Ecology, 74, 570-582.

Junta de CASTILla y León (2005). Castilla y León crece con el bosque. Consejería de Medio Ambiente, Junta de Castilla y León. 48 pp.

KIELY, G. (1999). Climate change in Ireland from precipitation and streamflow observation. Advances in Water Resources, 23: 141-151.

LABAJO, J.L. y PiORNO, A. (2001). Regionalisation of precipitation in Castilla and Leon (Spain). Analysis of its temporal behaviour. En Brunet, M. y López Bonillo, D. (Eds.): Detecting and Modelling Regional Climate Change. Springer. pp. 163-173.

Llorens, P., Poyatos, R., Rubio, C., Latron, J. y Gallart, F. (2005). El papel del bosque en los procesos hidrológicos. Ejemplos en las cuencas de investigación de Vallcebre (Prepirineo Catalán). Cuadernos de Investigación Geográfica, 31: 27-44.

Loaiciga, H. A., Valdés, J. B., Vogel, R., Garvey, J., Schwarz, H. (1996). Global warning and the hydrological cycle. Journal of Hydrology, 174: 83-127. 
MASACHS AlaVEDRA, V. (1948). El régimen de los ríos peninsulares. CSIC, Barcelona.

Mimikou, M.A., Baltas, E., VAranou, E. y PAntaZis, K. (2000). Regional impacts of climate change on water resources quantity and quality indicators. Journal of Hydrology, 234: 95-109.

Ministerio De Agricultura (1975). Inventario Forestal Nacional. Estimaciones comarcales y mapas, cuaderno $n^{\circ} 1$. Subdirección General de Protección de la Naturaleza. Madrid.

Ministerio de Agricultura, Pesca y Alimentación (1994). Segundo Inventario Forestal Nacional. Castilla y León: Ávila. Instituto Nacional para la Conservación de la Naturaleza-ICONA, Madrid. 202 pp + mapas.

Ministerio De Medio Ambiente (2000). Estrategia Forestal Española. Dirección General de Conservación de la Naturaleza, Madrid.

Milly, P.C.D., DunNe, K.A. y VeCChIA, A.V. (2005). Global pattern of trends in streamflow and water availability in a changing climate. Nature, 438/17: 347-350.

Morales, C., Ortega, M.T., Labajo, J.L. y Piorno, A. (2005). Recent trends and temporal behavior of thermal variables in the region of Castilla-León (Spain). Atmósfera, 18(2): 71-90.

OlIVER, J.F. (1980). Monthly precipitation distribution: a comparative index. Professional Geogr. 32: 300-309.

OSE (2006). Cambios de ocupación del suelo en España. Implicaciones para la sostenibilidad. Observatorio de la Sostenibilidad en España, Universidad de Alcalá. 485 pp.

PARry, M., PARrY, C. y LIVERMORE, M. (Eds.) (2000). Valoración de los efectos potenciales del Cambio Climático en Europa (Informe ACACIA de la Comisión Europea, resumen y conclusiones). Universidad de Castilla-La Mancha e Iberdrola. Toledo. 29 pp.

Pnuma (2000). Perspectivas del Medio Ambiente Mundial. Ediciones Mundi-Prensa, Madrid. 397 pp.

RAMBAL, S. (1987). Évolution de l'occupation des terres et ressources en eau en région Méditerranéenne karstique. Journal of Hydrology, 93: 339-357.

Ruiz DE LA TORRE, J. y CEBAllos, L. (1979). Árboles y arbustos de la España peninsular. ETS de Ingenieros de Montes, Fundación del Conde del Valle de Salazar. Madrid, $512 \mathrm{pp}$.

SCIAN, B. y DONNARI, M. (1997). Retrospective analysis of the palmer Drought Severity Index in the semiarid pampas region, Argentina. International Journal of Climatology, 17: 313-322. 
Shorthouse, C. y ARnell, N. (1999). The effects of climate variability on spatial characteristics of European river flows. Physics and Chemistry Earth, 24(1-2): 7-13.

SNEYERS, R. (1992). Use and misuse of statistical methods for detection of climatic change. In: Climate Change Detection Project, Report on the Informal Planning Meeting on Statistical Procedures for Climate Change Detection. WCDMP, (20); J76-J81.

Úbeda, X., Reina, L. y Sala, M. (1999). Cuantificación de la erosión en un camino forestal de un bosque típico mediterráneo. Norba, Revista de Geografía, 10: 185-196.

XU, C.Y. y Halldin, S. (1997). The effect of climate change on river flow and snow cover in the NOPEX area simulated by a single water balance model. Nordic Hydrology, 28: 273-282.

Yue, S., Pilon, P. y CaVAdias, G. (2002). Power of the Mann-Kendall and Spearman's rho tests for detecting monotonic trends in hydrological series. Journal of Hydrology, 259: 254-271.

ZHANG, L., DAWES, W.R. y WALKER, G.R. (2001). Response of mean annual evapotranspiration to vegetation changes at catchment scale. Water Resources Research, 37: 701-708. 